\title{
Exact tail asymptotics in bivariate scale mixture models
}

\author{
Enkelejd Hashorva
}

Received: 19 April 2010 / Revised: 3 February 2011 /

Accepted: 15 February 2011 / Published online: 11 March 2011

(C) Springer Science+Business Media, LLC 2011

\begin{abstract}
Let $(X, Y)=\left(R U_{1}, R U_{2}\right)$ be a given bivariate scale mixture random vector, with $R>0$ independent of the bivariate random vector $\left(U_{1}, U_{2}\right)$. In this paper we derive exact asymptotic expansions of the joint survivor probability of $(X, Y)$ assuming that $R$ has distribution function in the Gumbel max-domain of attraction, and $\left(U_{1}, U_{2}\right)$ has a specific local asymptotic behaviour around some absorbing point. We apply our results to investigate the asymptotic behaviour of joint conditional excess distribution and the asymptotic independence for two models of bivariate scale mixture distributions.
\end{abstract}

Keywords Tail asymptotics · Conditional excess distributions ·

Gumbel max-domain of attraction · Elliptically symmetric distributions ·

Dirichlet distributions · Residual tail dependence index · Davis-Resnick tail property

AMS 2000 Subject Classifications Primary-60F05; Secondary-60G70

\section{Introduction}

Let $(X, Y)$ be a bivariate random vector with stochastic representation

$$
(X, Y) \stackrel{d}{=}\left(R U_{1}, R U_{2}\right)
$$

\footnotetext{
E. Hashorva $(\bowtie)$

Department of Actuarial Science, Faculty of Business and Economics,

University of Lausanne, UNIL-Dorigny, 1015 Lausanne, Switzerland

e-mail: enkelejd.hashorva@unil.ch

E. Hashorva

Department of Mathematical Statistics and Actuarial Science,

University of Bern, Sidlerstrasse 5, 3012 Bern, Switzerland
} 
where $R>0$ is independent of the bivariate random vector $\left(U_{1}, U_{2}\right)$ ( $\stackrel{d}{=}$ stands for equality of distribution functions). The random vector $(X, Y)$ has a scale mixture distribution; a canonical example of such $(X, Y)$ is a bivariate spherical random vector with rotational invariant distribution function $(\mathrm{df})$ with $\left(U_{1}, U_{2}\right)$ uniformly distributed on the unit circle of $\mathbb{R}^{2}$. In this model (see Cambanis et al. 1981) the dependence between $U_{1}$ and $U_{2}$ is a functional one, namely $U_{1}^{2}+U_{2}^{2}=1$ almost surely, and

$$
\left(U_{1}, U_{2}\right) \stackrel{d}{=}\left(I_{1} W, I_{2} \sqrt{1-W^{2}}\right)
$$

with $I_{1}, I_{2} \in\{-1,1\}, W \in(0,1)$ almost surely, $W^{2}$ being beta distributed with parameters $\{1 / 2,1 / 2\}$, and $\boldsymbol{P}\left\{I_{1}=1\right\}=\boldsymbol{P}\left\{I_{2}=1\right\}=1 / 2$. Furthermore, $I_{1}, I_{2}, R, W$ are mutually independent.

Our main interest in this paper is the tail asymptotics of the joint survivor probability of $(X, Y)$. If $R$ is such that $R^{2}$ is chi-square distributed with 2 dof, then $X$ and $Y$ are independent Gaussian random variables with mean zero and variance 1 . For Gaussian random vectors the asymptotics of the joint survivor probability is investigated by many authors, see e.g., Berman (1962), Dai and Mukherjea (2001), or Lu and $\mathrm{Li}$ (2009). The elliptical model is obtained by extending Eq. 2 to

$$
\begin{aligned}
\left(U_{1}, U_{2}\right) & \stackrel{d}{=}\left(I_{1} W, I_{1} \rho W+I_{2} \rho_{*} \sqrt{1-W^{2}}\right), \quad \rho \in(-1,1), \\
\rho_{*} & :=\sqrt{1-\rho^{2}} .
\end{aligned}
$$

Hashorva (2007) generalises the known tail asymptotic results for Gaussian random vectors to the more general class of elliptically symmetric (for short elliptical) random vectors by exploiting the fact that the asymptotics of the joint survivor probability is determined by the asymptotics of the survivor function $\bar{F}:=1-F$ of the associated random radius $R$. Specifically, in the aforementioned paper the principal assumption is that $F$ is in the Gumbel max-domain of attraction (MDA), which means that for some positive scaling function $w$

$$
\lim _{x \rightarrow \infty} \frac{\bar{F}(x+t / w(x))}{\bar{F}(x)}=\exp (-t), \quad \forall t \in \mathbb{R} .
$$

As shown in Hashorva (2007) condition (4) is crucial when $(X, Y)$ is an elliptical random vector with stochastic representation (1) and $\left(U_{1}, U_{2}\right)$ satisfies Eq. 3. More specifically, for any $a \in(\rho, 1]$

$$
\boldsymbol{P}\{X>x, Y>a x\} \sim \frac{a_{\rho}^{2} \rho_{*}^{3}}{2 \pi(1-a \rho)(a-\rho)} \frac{1}{v\left(a_{\rho} x\right)} \bar{F}\left(a_{\rho} x\right),
$$

where

$$
a_{\rho}:=\rho_{*}^{-1} \sqrt{1-2 a \rho+a^{2}}>1, \quad v(x):=x w(x), \quad x \in \mathbb{R} .
$$


Throughout this paper $f(x) \sim g(x)$ means $\lim _{x \rightarrow \infty} f(x) / g(x)=1$, and Eq. 4 is abbreviated by $F \in G M D A(w)$.

As shown in Manner and Segers (2011), if $W^{2}$ is beta distributed with positive parameters $\alpha, \beta$ (its df is denoted by beta $(\alpha, \beta))$, then $(X, Y)$ is a generalised Dirichlet random vector. Hashorva (2009c) extends Eq. 5 for the class of Dirichlet random vectors. As indicated in Hashorva $(2009 \mathrm{a}, \mathrm{b})$ for certain asymptotic problems the distributional properties of $\left(U_{1}, U_{2}\right)$ do not need to be explicitly known.

A natural question that arises is that what models for the dependence between $U_{1}$ and $U_{2}$ lead to asymptotic results similar to Eq. 5? In this paper we answer the above question for two specific models: The first one is referred to as the unconstrained dependence model, or simply Model A. In that model we assume that $U_{1} \in(0,1]$ almost surely. Additionally, we impose a local asymptotic assumption on the behaviour of $\left(U_{1}, U_{2}\right)$ around some absorbing point, see Eq. 10 below.

The second model (or simply Model B) motivated by Eq. 3 is referred to as the functional dependence model where we assumes

$$
\left(U_{1}, U_{2}\right) \stackrel{d}{=}\left(I_{1} W, \rho I_{1} W+I_{2} z^{*}(W)\right), \quad \rho \in(-1,1),
$$

with $z^{*}$ some positive measurable function $I_{1}, I_{2} \in\{-1,1\}, W \in(0,1)$ almost surely, and $I_{1}, I_{2}, W$ are mutually independent. Model A differs substantially from Model B since $U_{1}$ and $U_{2}$ are not related to each other. Model B is a natural generalisation of the elliptical random vectors, however we do not impose distributional assumptions for our asymptotic treatment.

For both models we present several examples and provide some applications. Our first application establishes an asymptotic approximation of the joint conditional excess distribution. In the second application we discuss the Gumbel MDA of bivariate distributions related to Model B. In the third application we derive an explicit expression of the residual tail dependence index $\eta$ for bivariate scale mixture random vectors.

Organisation of the paper: In the next section we state our first result dealing with some general scale mixture bivariate random vectors which fall under Model A. We introduce in Section 3 some constrains on the dependence function of $\left(U_{1}, U_{2}\right)$ via Eq. 7, and then investigate the tail asymptotics of interest for Model B giving a generalisation of Eq. 5 in Theorem 3. The applications are presented in Section 4 followed by the proofs of all the results which are relegated to Section 5 .

\section{Tail asymptotics under unconstrained dependence}

Consider a bivariate scale mixture random vector $(X, Y)=\left(R U_{1}, R U_{2}\right)$, where $R$ has df $F$ (denote this $R \simeq F$ ). We assume throughout this paper that $F$ has an infinite upper endpoint satisfying Eq. 4 with some positive scaling function $w$. It is well-known (see e.g., Resnick 2008 or Falk et al. 2010) that $w$ can be defined asymptotically by

$$
w(x) \sim \frac{\bar{F}(x)}{\int_{x}^{\infty} \bar{F}(s) d s},
$$


where the integral mentioned above is finite, and further

$$
v(x):=x w(x) \rightarrow \infty, \quad x \rightarrow \infty .
$$

Given a constant $a \in(0,1]$ we investigate the asymptotics of

$$
p_{a, \delta, \eta}(x):=\boldsymbol{P}\{X>x[1+\delta / v(x)], Y>a x[1+\eta / v(x)]\}, \quad x \rightarrow \infty
$$

for $\delta, \eta \in[0, \infty)$. The reason for dealing with $p_{a, \delta, \eta}(x)$ is our interest concerning the approximation of the joint conditional excess distribution, see the first application in Section 4.

Throughout in the sequel we assume that $U_{1}$ is a bounded random variable. Without loss of generality we consider only the case $U_{1}$ has df with upper endpoint equal 1. This implies that $p_{a, \delta, \eta}(x) \leq \bar{F}(x)$ for any $x$ positive. For both Models A and B we show below that this upper bound is too crude; roughly speaking we show the asymptotic behaviour

$$
p_{a, \delta, \eta}(x) \sim \psi(x) \bar{F}(x),
$$

with $\psi$ some positive function decaying polynomially fast to 0 as $x \rightarrow \infty$.

In addition to the Gumbel MDA assumption in Eq. 4 we impose next a certain asymptotic behaviour of $\left(U_{1}, U_{2}\right)$ around $(1, a)$, namely for $\delta, \eta \in[0, \infty)$

$$
\begin{aligned}
\lim _{x \rightarrow \infty} \frac{\boldsymbol{P}\left\{U_{1}>1-(s-\delta) / x, U_{2}>a(1-(s-\eta) / x)\right\}}{\boldsymbol{P}\left\{U_{a}>1-1 / x\right\}} \\
=\xi_{a}(s, \delta, \eta), \quad s \in(0, \infty),
\end{aligned}
$$

with $\xi_{a}$ a positive measurable function and $U_{a}:=\min \left(U_{1}, U_{2} / a\right)$. Note that for $\delta \in[0, \eta]$

$$
\xi_{a}(s+\delta, 0, \eta-\delta)=\xi_{a}(s, \delta, \eta), \quad \forall s \in(0, \infty) .
$$

If $\delta=\eta=0$, then condition (10) reduces to

$$
\boldsymbol{P}\left\{U_{a}>1-s\right\}=s^{\gamma} L_{a}(s), \quad \xi_{a}(s, 0,0)=s^{\gamma}, \forall s>0
$$

for some $\gamma \in[0, \infty)$, with $L_{a}$ a positive measurable function such that $\lim _{s \downarrow 0} L_{a}(s) / L_{a}(t s)=1, \forall t>0$, i.e., $L_{a}$ is slowly varying; see Bingham et al. (1987), De Haan and Ferreira (2006) or Jessen and Mikosch (2006) for more details on regularly varying functions.

Theorem 1 Let $(X, Y)=\left(R U_{1}, R U_{2}\right)$ be a bivariate scale mixture random vector with $R \simeq F$ a positive random variable being independent of $\left(U_{1}, U_{2}\right)$, and let $a \in(0,1], \delta, \eta \in[0, \infty)$ be given constants. Suppose that $F$ has an infinite upper endpoint satisfying Eq. 4 with some positive scaling function $w$, and $U_{1} \in(0,1]$ has 
df with upper endpoint 1. Assume that Eq. 12 is satisfied with some function $L_{a}$ and $\gamma \in[0, \infty)$, and if $\delta+\eta>0$ suppose further that Eq. 10 holds. Then we have

$$
p_{a, \delta, \eta}(x) \sim J_{\delta, \eta} L_{a}(1 / v(x)) \frac{\bar{F}(x)}{(v(x))^{\gamma}}, \quad v(x):=x w(x),
$$

with

$$
J_{\delta, \eta}:=\int_{\delta}^{\infty} \xi_{a}(s, \delta, \eta) \exp (-s) d s \in(0, \infty)
$$

\section{Remarks}

(a) In view of Lemma 6.1 in Hashorva (2009b) for any $\lambda \in(1, \infty), c \in \mathbb{R}$ and $F$ as in Theorem 1 we have

$$
\lim _{x \rightarrow \infty} \frac{(v(x))^{c} \bar{F}(\lambda x)}{\bar{F}(x)}=0 .
$$

In fact Eq. 14 follows directly from Proposition 1.1 in Davis and Resnick (1988), see also Embrechts et al. (1997, p. 586), and A1. in Hashorva (2009c). We refer to Eq. 14 as the Davis-Resnick tail property.

Further we have the self-neglecting property of $w$, i.e.,

$$
\frac{w(x+t / w(x))}{w(x)} \sim 1
$$

holds locally uniformly for $t \in \mathbb{R}$. Refer to Reiss (1989), Embrechts et al. (1997), De Haan and Ferreira (2006), Resnick (2008), or Falk et al. (2010) for details on the Gumbel MDA.

(b) Under the assumptions of Theorem 1 applying Theorem 4.1 in Hashorva et al. (2010)

$$
p_{a, \delta, \eta}(x) \sim \boldsymbol{P}\left\{R W_{a}>x\right\}
$$

with $R$ independent of the random variable $W_{a}$ which is positive and satisfies

$$
\boldsymbol{P}\left\{W_{a}>1-1 / x\right\} \sim \frac{J_{\delta, \eta}}{\Gamma(\gamma+1)} L_{a}(1 / x) x^{-\gamma} .
$$

Furthermore, Eq. 13 holds locally uniformly for $\eta, \delta \in[0, \infty)$.

(c) By Eqs. 4, 13 and 15

$$
J_{\delta, \eta}=\exp (-\delta) J_{0, \eta-\delta}, \quad \forall \delta \in[0, \eta]
$$

which follows also directly by the definition on $J_{\delta, \eta}$ and Eq. 11 . Note that $J_{0,0}=$ $\Gamma(\gamma+1)$. 
We present next three illustrating examples.

Example 1 Let $U_{1}, U_{2}$ be two random variables taking values in $[0,1]$ such that $U_{2} \geq U_{1}$ almost surely. Suppose that $\boldsymbol{P}\left\{U_{1}>1-s\right\}=s^{\gamma} L(s), s \in(0,1)$ with $\gamma \in[0, \infty)$ and $L$ a slowly varying function at 0 . If $R$ is independent of $\left(U_{1}, U_{2}\right)$ satisfying the assumptions of Theorem 1 , we obtain

$$
\boldsymbol{P}\left\{R U_{1}>x\right\}=\boldsymbol{P}\left\{R U_{1}>x, R U_{2}>x\right\} \sim \Gamma(\gamma+1) L(1 / v(x)) \frac{\bar{F}(x)}{(v(x))^{\gamma}} .
$$

For $U_{1}^{2} \simeq \operatorname{bet} a(\alpha, \beta)$ the asymptotics in Eq. 17 is shown in Berman (1983). For the more general case that $U_{1}$ has a regularly varying survivor function see Theorem 3.1 in Hashorva et al. (2010).

Example 2 Let $S_{i} \simeq G_{i}, i=1,2$ be two independent random variables with values in $[0,1]$ such that

$$
\lim _{x \rightarrow \infty} \frac{\bar{G}_{i}(1-s / x)}{\bar{G}_{i}(1-1 / x)}=\gamma_{i}, \quad \forall s>0, i=1,2,
$$

with $\gamma_{i} \in[0, \infty)$. Let $\lambda_{1}, \lambda_{2} \in(0,1)$ be given constants with $\lambda_{1} \geq \lambda_{2}$, and set

$$
U_{i}:=\lambda_{i} S_{1}+\overline{\lambda_{i}} S_{2}, \quad \bar{\lambda}_{i}:=1-\lambda_{i}, \quad i=1,2
$$

For all $x$ large and $s>0$, for any $\delta, \eta \in[0, \infty)$ we may write (set $G_{2, x}(z):=$ $\left.G_{2}(1-z / x), s_{1}:=s-\delta, s_{2}:=s-\eta\right)$

$$
\begin{aligned}
\boldsymbol{P}\left\{U_{1}>1-s_{1} / x, U_{2}>1-s_{2} / x\right\}= & \bar{G}_{1}(1-1 / x) \bar{G}_{2}(1-1 / x) \\
& \times \int_{0}^{\infty} \frac{\boldsymbol{P}\left\{S_{1}>1-\left[s_{i}-\overline{\lambda_{i}} z\right] /\left(x \lambda_{i}\right), i=1,2\right\}}{\bar{G}_{1}(1-1 / x)} \\
& \times d G_{2, x}(z) / \bar{G}_{2}(1-1 / x) .
\end{aligned}
$$

By Eq. 18 for $s>\max (\delta, \eta)$ we obtain

$$
\begin{aligned}
\boldsymbol{P} & \left\{U_{1}>1-(s-\delta) / x, U_{2}>1-(s-\eta) / x\right\} \\
& \sim \tilde{\xi}(s, \delta, \eta) \bar{G}_{1}(1-1 / x) \bar{G}_{2}(1-1 / x),
\end{aligned}
$$

with

$$
\begin{aligned}
\tilde{\xi}(s, \delta, \eta):= & \gamma_{2} \int_{0}^{\infty}\left(\max \left(0, \min \left(\left[s-\delta-\overline{\lambda_{1}} z\right] / \lambda_{1},\left[s-\eta-\overline{\lambda_{2}} z\right] / \lambda_{2}\right)\right)\right)^{\gamma_{1}} \\
& \times z^{\gamma_{2}-1} d z .
\end{aligned}
$$


Note that for $s \in(0, \max (\delta, \eta)]$ Eq. 19 holds with $\tilde{\xi}(s, \delta, \eta)=0$, and

$$
\begin{aligned}
\tilde{\xi}(s, 0,0)= & {\left[\lambda_{1}^{-\gamma_{1}} \int_{0}^{1}\left[1-\overline{\lambda_{1}} t\right]^{\gamma_{1}} t^{\gamma_{2}-1} d t\right.} \\
& \left.+\lambda_{2}^{-\gamma_{1}} \int_{1}^{1 / \bar{\lambda}_{2}}\left[1-\overline{\lambda_{2}} t\right]^{\gamma_{1}} t^{\gamma_{2}-1} d t\right] s^{\gamma_{1}+\gamma_{2}}, \quad s>0 .
\end{aligned}
$$

Consequently, Eq. 10 holds with

$$
\xi_{1}(s, \delta, \eta)=\frac{\tilde{\xi}(s, \delta, \eta)}{\tilde{\xi}(s, 0,0)} \mathbf{1}(s>\max (\delta, \eta)), \quad s>0,
$$

where 1() is the indicator function. Thus with $R \simeq F$ such that $F \in G M D A(w)$ the result of Theorem 1 holds.

Example 3 Let $U_{i} \simeq G_{i}, i=1,2$ be two random variables with values in $[0,1]$. Suppose that for some $K \in[0,1)$

$$
\boldsymbol{P}\left\{U_{1}>x, U_{2}>y\right\}=\bar{G}_{1}(x) \bar{G}_{2}(y)\left[1+K \bar{G}_{1}(x) \bar{G}_{2}(y)\right], \quad \forall x, y \in[0,1] .
$$

The bivariate random vector $\left(U_{1}, U_{2}\right)$ possesses thus the Farlie-GumbelMorgenstern distribution, see e.g., Hashorva and Hüsler (1999). If Eq. 18 holds, then for any $\delta, \eta, s \in[0, \infty)$ we obtain

$$
\begin{aligned}
\boldsymbol{P} & \left\{U_{1}>1-(s-\delta) / x, U_{2}>1-(s-\eta) / x\right\} \\
& \sim(s-\delta)_{+}^{\gamma_{1}}(s-\eta)_{+}^{\gamma_{2}} \bar{G}_{1}(1-1 / x) \bar{G}_{2}(1-1 / x),
\end{aligned}
$$

with $(x)_{+}:=\max (x, 0), x \in \mathbb{R}$. Consequently, if the positive random variable $R \simeq$ $F$ is independent of $\left(U_{1}, U_{2}\right)$ and $F \in G M D A(w)$, then locally uniformly in $\delta, \eta$

$$
\begin{aligned}
p_{a, \delta, \eta}(x) \sim & {\left[\int_{0}^{\infty}(t-\delta)_{+}^{\gamma_{1}}(t-\eta)_{+}^{\gamma_{2}} \exp (-t) d t\right] } \\
& \times \bar{G}_{1}(1-1 / v(x)) \bar{G}_{2}(1-1 / v(x)) \bar{F}(x) .
\end{aligned}
$$

For any $a \in(0,1)$ we observe another asymptotic behaviour, namely if $G_{2}(a)$ is continuous at $a$ with $G_{2}(a) \in(0,1)$

$$
\boldsymbol{P}\left\{U_{1}>1-(s-\delta) / x, U_{2}>a(1-(s-\eta) / x)\right\} \sim(s-\delta)_{+}^{\gamma_{1}} \bar{G}_{1}(1-1 / x) \bar{G}_{2}(a)
$$

implying thus

$$
p_{a, \delta, \eta}(x) \sim \Gamma\left(\gamma_{1}+1\right) \exp (-\delta) \bar{G}_{2}(a) \bar{G}_{1}(1-1 / v(x)) \bar{F}(x) .
$$




\section{Tail asymptotics for functional dependence}

In this section we deal with bivariate scale mixture random vectors assuming that the dependence between the components is determined by some deterministic function. Explicitly, let $(X, Y)$ be a bivariate random vector with stochastic representation

$$
(X, Y) \stackrel{d}{=}\left(R I_{1} W, \rho R I_{1} W+R I_{2} z^{*}(W)\right), \quad \rho \in(-1,1),
$$

with $\left(I_{1}, I_{2}\right), R>0, W \in(0,1)$ mutually independent, and $z^{*}:[0,1] \rightarrow[0,1]$ a positive measurable function.

We assume that the df $F$ of $R$ has an infinite upper endpoint, and the df of $W$ has upper endpoint equal 1 . In the sequel $I_{1}, I_{2}$ take values in $\{-1,1\}$ with $\boldsymbol{P}\left\{I_{1}=I_{2}=1\right\} \in(0,1]$; we allow $I_{1}$ and $I_{2}$ to be dependent.

The random vector $(X, Y)$ is a scale mixture random vector for which the dependence of the components is being determined by $\rho, z^{*}$ and the random variables $R, W, I_{i}, i=1,2$. We refer to the implied dependence of the components as the functional dependence. Note in passing that if

$$
W^{2} \simeq \operatorname{beta}(1 / 2,1 / 2), \quad z^{*}(x)=\sqrt{1-x^{2}}, \quad x \in[0,1],
$$

and $I_{1}, I_{2}$ are independent with $\boldsymbol{E}\left\{I_{1}\right\}=\boldsymbol{E}\left\{I_{2}\right\}=0$, then $(X, Y)$ is a bivariate elliptical random vector.

Generally speaking, it turns out that under Eq. 22 the local asymptotics of the probability density function (pdf) of $W$ is important. More precisely, we shall derive an asymptotic expansion of

$$
p_{a}(x):=\boldsymbol{P}\{X>x, Y>a x\}, \quad a \in(0,1], x>0
$$

requiring further that for some constant $a_{\rho} \in(1, \infty)$

$$
\boldsymbol{P}\left\{W-1 / a_{\rho} \in\left(K_{1} s, K_{2} s\right)\right\} \sim L_{K_{1}, K_{2}}(s) s^{\gamma_{a}}, \quad \gamma_{a} \in[0, \infty)
$$

holds for all $s>0$ small with $K_{1}<K_{2}, K_{1}, K_{2} \in \mathbb{R}$ some given constants such that $L_{K_{1}, K_{2}}$ is a locally bounded slowly varying function at 0 . Additionally, we need to impose a local asymptotic condition on the inverse of the transformation $z$ (see below (Eq. 24)).

We state first the result for $p_{a}(x)$.

Theorem 2 Let $(X, Y), R \simeq F, \rho \in(-1,1)$ be a bivariate random vector with stochastic representation (Eq. 22), where $z^{*}:[0,1] \rightarrow[0,1]$ is a positive measurable function, and let $a \in(0,1], a_{\rho} \in(1, a /|\rho|)$ be given constants. Suppose that for some $\varepsilon \in(0,1)$ the function $z(x):=\rho x+z^{*}(x), x \in[0,1]$ is decreasing in 
$V_{\varepsilon}:=\left[1 / a_{\rho}-\varepsilon, 1 / a_{\rho}+\varepsilon\right]$ and $z(x) \leq a / a_{\rho}, \forall x \in\left(1 / a_{\rho}, 1\right]$. Assume further that the inverse $z_{\varepsilon}$ of $z$ in $V_{\varepsilon}$ satisfies

$$
z_{\varepsilon}\left(a / a_{\rho}-1 / x\right)-1 / a_{\rho} \sim \frac{c}{x}
$$

locally uniformly for $x>0$ with $c \in(0, \infty)$. If $F \in G M D A(w)$, and Eq. 23 is satisfied with $K_{1}:=-1 / a_{\rho}, K_{2}:=c a / a_{\rho}$, then $a_{\rho}$ is unique and

$$
p_{a}(x) \sim \boldsymbol{P}\left\{I_{1}=I_{2}=1\right\} \Gamma\left(\gamma_{a}+1\right) L_{K_{1}, K_{2}}\left(1 / v\left(x_{*}\right)\right) \frac{\bar{F}\left(x_{*}\right)}{\left(v\left(x_{*}\right)\right)^{\gamma_{a}}},
$$

where $x_{*}:=a_{\rho} x, v(x):=x w(x), x>0$.

\section{Remarks}

(a) If $W$ in Eq. 22 possesses a positive pdf $h$ continuous at $1 / a_{\rho}$, then under the assumptions of Theorem 2 the asymptotics in Eq. 23 holds for any $K_{1}<K_{2}$, with $\gamma_{a}=1$ and

$$
L_{K_{1}, K_{2}}(u)=\left(K_{2}-K_{1}\right) h\left(1 / a_{\rho}\right), \quad u>0 .
$$

(b) In view of Eq. $14 p_{a}(x)$ given by Eq. 25 converges faster to 0 than $\bar{F}(x)$. In fact for any constant $\mu>0$ we have (recall Eq. 9 and the Davis-Resnick tail property)

$$
\lim _{x \rightarrow \infty} \frac{p_{a}(x)}{(v(x))^{\mu} \bar{F}(x)}=0
$$

(c) As it can be seen from the proof of Theorem 2 the local behaviour of $z$ at $1 / a_{\rho}$ is crucial. Another tractable specification for the function $z$ is to assume that it is increasing in $\left(1 / a_{\rho}-\varepsilon, 1 / a_{\rho}\right)$ and decreasing in $\left(1 / a_{\rho}, 1 / a_{\rho}+\varepsilon\right)$ with $1 / a_{\rho}$ a local maximum. In this case we can still find the asymptotics of $p_{a}(x)$, provided that additionally $z_{\varepsilon}^{-}$and $z_{\varepsilon}^{+}$are the inverses of $z$ in $\left(1 / a_{\rho}-\varepsilon, 1 / a_{\rho}\right)$ and $\left(1 / a_{\rho}, 1 / a_{\rho}+\varepsilon\right)$, respectively satisfying

$$
z_{\varepsilon}^{ \pm}\left(a / a_{\rho}-1 / x\right)-1 / a_{\rho} \sim-\frac{c_{ \pm}}{x}, \quad c_{ \pm} \in(0, \infty)
$$

locally uniformly for $x>0$.

In the setting of Model B we shall approximate $p_{a, \delta, \eta, \rho}(x)$ defined by

$$
p_{a, \delta, \eta, \rho}(x):=\boldsymbol{P}\left\{X>x\left[1+\delta / v\left(a_{\rho} x\right)\right], Y>\operatorname{ax}\left[1+\eta / v\left(a_{\rho} x\right)\right]\right\}, \quad x>0,
$$

with $\delta, \eta \in[0, \infty)$ and $a_{\rho} \in(1, a /|\rho|)$. 
Theorem 3 Under the assumptions and notation of Theorem 2, if further $F$ has pdf $f$ which is positive and continuous at $1 / a_{\rho}$, then $a_{\rho}$ is unique. If also

$$
z_{\varepsilon}\left(a / a_{\rho}+1 / x\right)-1 / a_{\rho} \sim-\frac{c}{x}
$$

locally uniformly for $x>0$, then for any $\eta, \delta \in[0, \infty)$

$$
p_{a, \delta, \eta, \rho}(x) \sim \boldsymbol{P}\left\{I_{1}=I_{2}=1\right\} \frac{h\left(1 / a_{\rho}\right)}{a_{\rho}}(c a+1) \exp \left(-\frac{\delta+c a \eta}{c a+1}\right) \frac{\bar{F}\left(x_{*}\right)}{v\left(x_{*}\right)}
$$

holds locally uniformly for $\delta, \eta \in[0, \infty)$.

We present next two examples.

Example 4 Let $(X, Y)$ be a bivariate scale mixture random vector with stochastic representation (Eq. 22) where $\rho=0$. Define next

$$
z^{*}(x)=z(x)=\left(1-|x|^{p}\right)^{1 / p}, \quad p \in(0, \infty), x \in[-1,1],
$$

where $z$ has the inverse function $z^{-1}(y)=\left(1-y^{p}\right)^{1 / p}, y \in[0,1]$. For any $a \in(0,1]$ the equation

$$
z^{-1}(a / s)=1 / s, \quad s \in(1, \infty)
$$

has the unique solution $a_{\rho}=\left(1+a^{p}\right)^{1 / p} \in(1, \infty)$. Furthermore Eqs. 24 and 27 hold with $c=a^{p-1}$.

Let $W>0$ with pdf $h$ being further independent of the positive random variable $R \simeq F$. If $F \in G M D A(w)$, then by Theorem 3

$$
p_{a, \delta, \eta, \rho}(x) \sim \boldsymbol{P}\left\{I_{1}=I_{2}=1\right\} a_{\rho}^{p-2} h\left(1 / a_{\rho}\right) \exp \left(-\frac{\delta+a^{p} \eta}{1+a^{p}}\right) \frac{\bar{F}\left(a_{\rho} x\right)}{x w\left(a_{\rho} x\right)} .
$$

Note that when $\left(R I_{1} W, I_{2} R z^{*}(W)\right)$ is a generalised symmetrised Dirichlet random vector, then $I_{1}, I_{2}, R, W$ are independent and $W$ possesses the pdf $h(x)=$ $p x^{p-1} g\left(x^{p}\right)$, with $g$ the pdf of beta $(\alpha, \beta)$, see Fang and Fang (1990).

Example 5 Under the setup of Example 4 redefine $z^{*}$ as

$$
z^{*}(x):=\rho_{*} \sqrt{1-x^{2}}, \quad z(x):=\rho x+z^{*}(x), \quad \rho, x \in(-1,1), \quad \rho_{*}:=\sqrt{1-\rho^{2}} .
$$

First note that $z(\rho)=1$ is the maximal value of $z(x)$ for any $x \in[-1,1]$. Hence in order to apply Eq. 28 necessarily $a \in(\rho, 1]$. The assumptions of Theorem 2 are satisfied for $a_{\rho}=\rho_{*}^{-1} \sqrt{1-2 a \rho+a^{2}}$, and Eq. 24 is satisfied for $c=(a-\rho) /(1-$ $a \rho) \in(0, \infty)$. Note further that $a_{\rho}<a /|\rho|$ and also Eq. 27 holds. In view of Eq. 28 
we obtain

$$
\begin{aligned}
p_{a, \delta, \eta, \rho}(x) \sim & \boldsymbol{P}\left\{I_{1}=I_{2}=1\right\} \frac{\rho_{*}^{2} h\left(1 / a_{\rho}\right)}{1-a \rho} \frac{\bar{F}\left(a_{\rho} x\right)}{x w\left(a_{\rho} x\right)} \\
& \times \exp \left(-\frac{a^{2} \eta+\delta-a \rho(\eta+\delta)}{\rho_{*}^{2} a_{\rho}^{2}}\right) .
\end{aligned}
$$

In the special case that $W^{2} \simeq \operatorname{beta}(1 / 2,1 / 2)$ and $\boldsymbol{P}\left\{I_{i}=1\right\}=1 / 2, i=1,2$ with $I_{1}, I_{2}$ independent we have

$$
h\left(1 / a_{\rho}\right)=\frac{2 a_{\rho}}{\pi \sqrt{a_{\rho}^{2}-1}}=\frac{2 a_{\rho}\left(1-\rho^{2}\right)}{a-\rho} .
$$

Consequently Eq. 30 reduces to Eq. 5 for $\delta=\eta=0$.

\section{Applications}

Let $(X, Y)$ be a given bivariate random vector. For some high threshold $x$ the approximation of the joint conditional excess random vector

$$
\left(X^{[x]}, Y^{[a x]}\right):=(X-x, Y-a x) \mid X>x, Y>a x, \quad x \in(0, \infty), \quad a \in(0,1]
$$

is of some interest in statistical applications if in particular suitable norming constants can be found so that the df of $\left(X^{[x]}, Y^{[a x]}\right)$ can be approximated by some known df, see De Haan and Ferreira (2006), Reiss and Thomas (2007) and Falk et al. (2010). Another interesting problem of the bivariate extreme value theory is the asymptotic independence of $X$ and $Y$. When $X$ and $Y$ are asymptotically independent an interesting topic also for application (see e.g., De Haan and Ferreira 2006; Reiss and Thomas 2007; or Peng 1998, 2008, 2010) is the estimation of the residual dependence index $\eta$. In our last application we give an explicit formula for $\eta$.

\subsection{Asymptotics of conditional excess distribution}

We start by considering Model A as in Section 2. For any $s, t$ positive and some positive scaling function $w$ we have

$$
\boldsymbol{P}\left\{X^{[x]}>s / w(t), Y^{[a x]}>t / w(t)\right\}=\frac{p_{a, s, t}(x)}{p_{a, 0,0}(x)}, \quad x>0 .
$$

Under the assumptions of Theorem 1

$$
\frac{p_{a, s, t}(x)}{p_{a, 0,0}(x)} \sim \frac{J_{s, t}}{J_{0,0}}, \quad x \rightarrow \infty,
$$


where $J_{s, t}$ depends on the limit function $\xi_{a}$. Denote by $\left(E_{1}, E_{2}\right)$ a bivariate random vector with positive components and survivor function given by $J_{s, t} / J_{0,0}, s, t \in$ $(0, \infty)$. Then the above asymptotics can be cast into joint convergence in distributions. Specifically, if $\left(X_{n}, Y_{n}\right), n \geq 1$ is a sequence of bivariate random vectors defined in the same probability space such that $\left(X_{n}, Y_{n}\right) \stackrel{d}{=}\left(X^{[n]}, Y^{[a n]}\right), n \geq 1$, then we have the convergence in distributions

$$
\left(g(n) X^{[n]}, g(n) Y^{[a n]}\right) \stackrel{d}{\rightarrow}\left(E_{1}, E_{2}\right), \quad n \rightarrow \infty
$$

where the scaling function $g$ equals $w$. The limiting random vector has df which clearly depends on $\xi_{a}$. Further, $E_{1}$ and $E_{2}$ can be dependent, for instance in the setup of Example 3 taking $a=1$.

Assume next that $(X, Y), a, a_{\rho}, \rho$ satisfy the assumptions of Theorem 3. For any $s, t$ positive Eq. 28 implies

$$
\frac{\boldsymbol{P}\left\{X>x+s / w\left(a_{\rho} x\right), Y>a x+t / w\left(a_{\rho} x\right)\right\}}{\boldsymbol{P}\{X>x, Y>a x\}} \sim \exp \left(-s D_{a, c}-t D_{a, c}^{*}\right),
$$

where

$$
D_{a, c}:=\frac{a_{\rho}}{c a+1}, \quad D_{a, c}^{*}:=\frac{a_{\rho} c}{c a+1} .
$$

Consequently, Eq. 31 holds with

$$
g(x)=w\left(a_{\rho} x\right), \quad x>0
$$

and $E_{1}, E_{2}$ two independent exponential random variables with mean $1 / D_{a, c}$ and $1 / D_{a, c}^{*}$, respectively.

Under the setup of Example 5

$$
D_{a, c}=\frac{1-a \rho}{a_{\rho}\left(1-\rho^{2}\right)}, \quad D_{a, c}^{*}=\frac{a-\rho}{a_{\rho}\left(1-\rho^{2}\right)}, \quad \rho \in(-1,1) .
$$

Consequently, Eq. 31 holds in the special case that $\left(U_{1}, U_{2}\right)$ is uniformly distributed on the unit circle of $\mathbb{R}^{2}$.

\subsection{Asymptotic independence and MDA}

A common measure of the asymptotic dependence between $(X, Y)$ is the tail dependence function

$$
\lim _{x \rightarrow \infty} \frac{\boldsymbol{P}\left\{G_{1}(X)>1-s / x, G_{2}(Y)>1-t / x\right\}}{\min \left(\boldsymbol{P}\left\{G_{1}(X)>1-s / x\right\}, \boldsymbol{P}\left\{G_{2}(Y)>1-t / x\right\}\right)}=: l(s, t), \quad s, t \in(0, \infty)
$$

(when it exists) where $G_{1}, G_{2}$ are the distribution functions of $X$ and $Y$, respectively. If $l(1,1)=0$, then we say that $X$ and $Y$ are asymptotically independent. 
See for instance De Haan and Ferreira (2006), Reiss and Thomas (2007), Hüsler and Li (2009), Peng (2010), Das and Resnick (2011) and Haug et al. (2011) for more details concerning the modelling of asymptotic independence in the context of extreme values.

We discuss briefly the asymptotic independence for scale mixture distributions with $\left(U_{1}, U_{2}\right)$ specified by Model A. It can be seen by Example 1 that for particular $\left(U_{1}, U_{2}\right)$ the dependence function $l(s, t)$ can be positive, thus asymptotic independence does not hold. However, under the setup of Example 2, Eq. 21 implies $l(s, t)=0, \forall s, t \in(0, \infty)$. Consequently, $X$ and $Y$ are asymptotically independent with df in the Gumbel MDA.

In the framework of Model B we consider $(X, Y)$ with stochastic representation (Eq. 22) where $\rho \in[0,1)$. The case $\rho \in(-1,0)$ follows with similar arguments, therefore omitted here. Next, we specify the asymptotic behaviour of $W$ and $z(W)$. Assume that for some $\gamma_{1}, \gamma_{2} \in[0, \infty)$

$$
\lim _{x \rightarrow \infty} \frac{\boldsymbol{P}\{W>1-s / x\}}{\boldsymbol{P}\{W>1-1 / x\}}=s^{\gamma_{1}}, \quad \lim _{x \rightarrow \infty} \frac{\boldsymbol{P}\{z(W)>1-s / x\}}{\boldsymbol{P}\{z(W)>1-1 / x\}}=s^{\gamma_{2}}, \quad \forall s>0 .
$$

As in Example 1, applying Eq. 17 we obtain

$$
\boldsymbol{P}\{R W>x\} \sim \Gamma\left(\gamma_{1}+1\right) \boldsymbol{P}\{W>1-1 / v(x)\} \bar{F}(x)
$$

and

$$
\boldsymbol{P}\{R z(W)>x\} \sim \Gamma\left(\gamma_{2}+1\right) \boldsymbol{P}\{z(W)>1-1 / v(x)\} \bar{F}(x) .
$$

Suppose that $z^{*}(x) \leq b<1, \forall x \in[0,1]$, and set $z(x):=\rho x+z^{*}(x)$. Applying Eq. 14 we obtain

$$
\boldsymbol{P}\{Y>x\} \sim \boldsymbol{P}\left\{I_{1}=1, I_{2}=1\right\} \boldsymbol{P}\{R z(W)>x\}
$$

and

$$
\boldsymbol{P}\{X>x\} \sim \boldsymbol{P}\left\{I_{1}=1\right\} \Gamma\left(\gamma_{1}+1\right) \boldsymbol{P}\{W>1-1 / v(x)\} \bar{F}(x) .
$$

Consequently, by Eq. 15 both $X$ and $Y$ have distribution functions in the Gumbel MDA with the same scaling function $w$. Let $b_{i}(x), i=1,2$ be defined asymptotically by

$$
b_{i}(x):=G_{i}^{-1}(1-1 / x), \quad x>1,
$$

where $G_{i}^{-1}$ is the generalised inverse of $G_{i}, i=1,2$. In view of Eq. 14 we have

$$
\lim _{x \rightarrow \infty} \frac{b_{1}(x)}{b_{2}(x)}=1 .
$$

Furthermore (see e.g., Falk et al. 2010)

$$
w\left(b_{i}(x)\right)\left[G_{i}^{-1}(1-s / x)-b_{i}(x)\right]=-\ln s, \quad \forall s \in(0, \infty) .
$$


If $X, Y$ are such that the conditions of Theorem 2 hold, then comparing the asymptotics of $\boldsymbol{P}\left\{X>b_{1}(x / s), Y>b_{2}(x / t)\right\}$ and $\boldsymbol{P}\left\{X>b_{1}(x / s)\right\}, \boldsymbol{P}\left\{Y>b_{2}(x / t)\right\}$ utilising further Eqs. 14 and 32 we obtain $l(s, t)=0, \forall s, t \in(0, \infty)$. Consequently, $X$ and $Y$ are asymptotically independent.

\subsection{Residual tail dependence}

Modeling of the asymptotic dependence is often done in the framework of copulas, see e.g., De Haan et al. (2008), Li and Peng (2009) and Peng and Qi (2011). For $X, Y$ with asymptotically independent components it is of some interest to quantify the asymptotic independence in terms of some indices. Let $G_{1}, G_{2}$ be the distribution functions of $X$ and $Y$, respectively. One successful approach to model the asymptotic independence is the estimation of the residual dependence index $\eta \in(0,1]$ (see e.g., Peng 1998, 2008, 2010; De Haan and Ferreira 2006; Reiss and Thomas 2007; Falk et al. 2010; or Hashorva 2010). So if for some $x, y$ positive

$$
S_{u}(x, y):=\frac{\boldsymbol{P}\left\{G_{1}(X)>1-x / u, G_{2}(Y)>1-y / u\right\}}{\boldsymbol{P}\left\{G_{1}(X)>1-1 / u, G_{2}(Y)>1-1 / u\right\}} \rightarrow S(x, y), \quad u \rightarrow \infty,
$$

then for any $c>0$

$$
S(c x, c y)=c^{1 / \eta} S(x, y),
$$

where $S_{u}(1,1)$ is regularly varying with index $-1 / \eta$.

$\eta$ for Model A

Example 1 shows that the asymptotic independence is not always observed for our first dependence model. We consider next the setup of Example 2 which exhibits asymptotic independence calculating $\eta$ for that example. Since $\lim _{u \rightarrow \infty} b_{1}(u)=$ $\lim _{u \rightarrow \infty} b_{2}(u)=\infty$, by Eqs. 21 and 33 for any $x, y \in(0,1]$ we obtain

$$
\begin{aligned}
& \lim _{u \rightarrow \infty} \frac{S_{u}(x, y)}{S_{u}(1,1)} \\
& =\lim _{u \rightarrow \infty} \frac{\boldsymbol{P}\left\{X>b_{1}(u)-\ln x / w\left(b_{1}(u)\right), Y>b_{2}(u)-\ln y / w\left(b_{2}(u)\right)\right\}}{\boldsymbol{P}\left\{X>b_{1}(u), Y>b_{2}(u)\right\}} \\
& =\frac{1}{\Gamma\left(\gamma_{1}+\gamma_{2}+1\right)} \int_{0}^{\infty}(t+\ln x)_{+}^{\gamma_{1}}(t+\ln y)_{+}^{\gamma_{2}} \exp (-t) d t
\end{aligned}
$$

implying that $\eta=1$.

\section{$\eta$ for Model $B$}

Let $(X, Y)$ be as in our second application satisfying further the assumptions of Theorem 3. Since $X, Y$ are asymptotically independent we calculate next $\eta$ assuming that the scaling function $w$ satisfies

$$
\lim _{u \rightarrow \infty} \frac{w(c u)}{w(u)}=c^{\lambda-1}, \quad \forall c \in(0, \infty)
$$


with $\lambda \in[0, \infty)$. Thus $w(x)=x^{\lambda-1} L(x)$ with $L$ a positive slowly varying function at infinity. If $\lambda=0$ suppose further that $\lim _{u \rightarrow \infty} L(u)=\infty$.

Case $\lim \sup _{u \rightarrow \infty} w(u) \in[0, \infty)$ In view of Eq. 32 for any $y \in(0, \infty)$

$$
b_{2}(u)-\frac{\ln y}{w\left(b_{2}(u)\right)}=b_{1}(u)-(1+o(1)) \frac{\ln y}{w\left(b_{1}(u)\right)}, \quad u \rightarrow \infty .
$$

Since $\lim _{u \rightarrow \infty} b_{1}(u)=\infty$, Eqs. 34 and 35 imply for any $x, y \in(0,1)($ set $\tau(u):=$ $\left.1 / w\left(\alpha_{\rho} b_{1}(u)\right)\right)$

$$
\begin{aligned}
& \lim _{u \rightarrow \infty} \frac{S_{u}(x, y)}{S_{u}(1,1)} \\
& =\lim _{u \rightarrow \infty} \frac{\boldsymbol{P}\left\{X>b_{1}(u)-\alpha_{\rho}^{\lambda-1} \tau(u) \ln x, Y>b_{1}(u)-(1+o(1)) \alpha_{\rho}^{\lambda-1} \tau(u) \ln y\right\}}{\boldsymbol{P}\left\{X>b_{1}(u), Y>b_{1}(u)+o(1) \tau(u)\right\}} \\
& =\exp \left(\alpha_{\rho}^{\lambda-1}\left(D_{a, c} \ln x+D_{a, c}^{*} \ln y\right)\right) .
\end{aligned}
$$

Consequently, since further $\alpha_{\rho}>1$

$$
\eta=\frac{\alpha_{\rho}^{1-\lambda}}{D_{1, c}+D_{1, c}^{*}}=\alpha_{\rho}^{-\lambda} \in(0,1]
$$

with $\eta=1$ only when $\lambda=0$.

Case $\lim _{u \rightarrow \infty} w(u)=\infty$ In order to calculate $\eta$ we assume further

$$
\lim _{u \rightarrow \infty} w\left(b_{2}(u)\right)\left[b_{2}(u)-b_{1}(u)\right]=\xi \in \mathbb{R} .
$$

As above for any $x, y \in(0,1)$ as $u \rightarrow \infty$ we obtain

$$
\begin{aligned}
& \lim _{u \rightarrow \infty} \frac{S_{u}(x, y)}{S_{u}(1,1)} \\
& =\lim _{u \rightarrow \infty} \frac{\boldsymbol{P}\left\{X>b_{1}(u)-\alpha_{\rho}^{\lambda-1} \tau(u) \ln x, Y>b_{1}(u)-(1+o(1)) \alpha_{\rho}^{\lambda-1} \tau(u)(\ln y+\xi)\right\}}{\boldsymbol{P}\left\{X>b_{1}(u), Y>b_{1}(u)+(1+o(1)) \alpha_{\rho}^{\lambda-1} \xi \tau(u)\right\}} \\
& =\exp \left(\alpha_{\rho}^{\lambda-1}\left(D_{a, c} \ln x+D_{a, c}^{*} \ln y\right)\right) .
\end{aligned}
$$

Hence again $\eta=\alpha_{\rho}^{-\lambda} \in(0,1]$, with $\eta=1$ only for $\lambda=0$. 


\section{Proofs}

Proof of Theorem 1 Since $R$ is independent of the bivariate random vector $\left(U_{1}, U_{2}\right)$, and $U_{1} \leq 1$ almost surely for any $\delta, \eta \in[0, \infty)$ we have

$$
\begin{aligned}
p_{a, \delta, \eta}(x)= & \boldsymbol{P}\left\{R U_{1}>x(1+\delta / v(x)), R U_{2}>\operatorname{ax}(1+\eta / v(x))\right\} \\
= & \int_{x}^{\infty} \boldsymbol{P}\left\{U_{1}>x(1+\delta / v(x)) / r, U_{2}>\operatorname{ax}(1+\eta / v(x)) / r\right\} d F(r), \\
& \forall x>0 .
\end{aligned}
$$

Let $\varepsilon$ be a positive constant. The assumption (Eq. 12) implies that for any $c \in(1,1+$ $\varepsilon$ ) we have $\boldsymbol{P}\left\{U_{1}>1 / c, U_{2}>a / c\right\} \in(0,1)$. In view of Eq. 14

$$
\lim _{x \rightarrow \infty} \frac{\bar{F}(\alpha x)}{\bar{F}(x)}=0, \quad \forall \alpha \in(1, \infty)
$$

hence we obtain $\left(\operatorname{set} F_{x}(s):=F(s / w(x)+x)\right)$

$$
\begin{aligned}
p_{a, \delta, \eta}(x) & \sim \int_{x(1+\delta / v(x))}^{c x} \boldsymbol{P}\left\{U_{1}>x(1+\delta / v(x)) / r, U_{2}>\operatorname{ax}(1+\eta / v(x)) / r\right\} d F(r) \\
& =\int_{\delta}^{(c-1) v(x)} q_{\delta, \eta}(s, v(x)) d F_{x}(s),
\end{aligned}
$$

with

$$
q_{\delta, \eta}(s, v(x)):=\boldsymbol{P}\left\{U_{1}>\frac{1+\delta / v(x)}{1+s / v(x)}, U_{2}>\frac{a(1+\eta / v(x))}{1+s / v(x)}\right\}, \quad s \geq \delta .
$$

Condition (10) implies

$$
\frac{q_{\delta, \eta}(s, v(x))}{\boldsymbol{P}\left\{U_{a}>1-1 / x\right\}} \rightarrow \xi_{a}(s, \delta, \eta), \quad x \rightarrow \infty
$$

locally uniformly $s \geq \delta$. Hence in view of Eqs. 4 and 9 Fatou's Lemma yields

$$
\liminf _{x \rightarrow \infty} \frac{1}{\bar{F}(x)} \int_{\delta}^{(c-1) v(x)} \frac{q_{\delta, \eta}(s, v(x))}{\boldsymbol{P}\left\{U_{a}>1-1 / x\right\}} d F_{x}(s) \geq \int_{\delta}^{\infty} \xi_{a}(s, \delta, \eta) \exp (-s) d s .
$$

When $\delta=\eta=0$, by Eq. $12 q_{\delta, \eta}(s, 1)$ is regularly varying at 0 . Utilising further Potter's bounds (see e.g., De Haan and Ferreira 2006) for the integrand and utilising Lemma 7.5 and 7.7 in Hashorva (2007) we obtain

$$
\limsup _{x \rightarrow \infty} \frac{1}{\bar{F}(x)} \int_{\delta}^{(c-1) v(x)} \frac{q_{0,0}(s, v(x))}{\boldsymbol{P}\left\{U_{a}>1-1 / x\right\}} d F_{x}(s) \leq J_{0,0},
$$


and further

$$
\lim _{M \rightarrow \infty} \frac{1}{\bar{F}(x)} \int_{M}^{(c-1) v(x)} \frac{q_{0,0}(s, v(x))}{\boldsymbol{P}\left\{U_{a}>1-1 / x\right\}} d F_{x}(s)=0
$$

Since for any $s \geq \delta$

$$
q_{\delta, \eta}(s, v(x)) \leq q_{0,0}(s, v(x)), \quad \delta, \eta \geq 0
$$

this implies

$$
\lim _{M \rightarrow \infty} \frac{1}{\bar{F}(x)} \int_{M}^{(c-1) v(x)} \frac{q_{\delta, \eta}(s, v(x))}{\boldsymbol{P}\left\{U_{a}>1-1 / x\right\}} d F_{x}(s)=0,
$$

hence the general case with $\delta, \eta \in[0, \infty)$ is established by utilising further Eqs. 10, 38 , and thus the proof is complete.

\section{Proof of Theorem 2 Define}

$$
x_{*}:=a_{\rho} x, \quad v(x)=x w(x), \quad F_{x}(s):=F\left(x_{*}\left[1+s / v\left(x_{*}\right)\right]\right), \quad s \in \mathbb{R}, x>0 .
$$

By the independence of $I_{1}, I_{2}$ and $R W$ we may write for any $x>0$

$$
\begin{aligned}
p_{a}(x)= & \boldsymbol{P}\left\{R W>x, \rho R W-z^{*}(W)>a x\right\} \boldsymbol{P}\left\{I_{1}=1, I_{2}=-1\right\} \\
& +\boldsymbol{P}\{R W>x, R z(W)>a x\} \boldsymbol{P}\left\{I_{1}=1, I_{2}=1\right\} \\
= & : C_{1} \boldsymbol{P}\left\{I_{1}=1, I_{2}=-1\right\}+C_{2} \boldsymbol{P}\left\{I_{1}=1, I_{2}=1\right\} .
\end{aligned}
$$

If $\rho \leq 0$, then the fact that $z^{*}$ is non-negative implies $p_{a}(x)=C_{2}$. When $\rho \in(0,1)$ by the assumption $a / \rho>a_{\rho}$

$$
C_{1} \leq \boldsymbol{P}\{R W>a / \rho x\} \leq \boldsymbol{P}\{R>a / \rho x\}=\bar{F}(a / \rho x) .
$$

Since $z(s) \leq a / a_{\rho}$ for any $s \in\left[1 / a_{\rho}, 1\right]$ we have

$$
\begin{aligned}
\boldsymbol{P}\{W>x / r, z(W)>a x / r\} & \leq \boldsymbol{P}\left\{W>1 / a_{\rho}, z(W)>a x / r\right\} \\
& \leq \boldsymbol{P}\left\{W>1 / a_{\rho}, z(W)>a / a_{\rho}\right\} \\
& =0, \quad \forall x, r>0, x \leq r \leq a_{\rho} x .
\end{aligned}
$$

Hence for any $\varepsilon \in(0,1)$

$$
\begin{aligned}
C_{2}= & \int_{a_{\rho} x}^{\left(a_{\rho}+\varepsilon\right) x} \boldsymbol{P}\{W>x / r, z(W)>a x / r\} d F(r) \\
& +\int_{\left(a_{\rho}+\varepsilon\right) x}^{\infty} \boldsymbol{P}\{W>x / r, z(W)>a x / r\} d F(r) .
\end{aligned}
$$


Since (by the assumption) the function $z$ is decreasing and possesses an inverse function $z_{\varepsilon}$ in $\left[1 / a_{\rho}-\varepsilon, 1 / a_{\rho}+\varepsilon\right]$ for some given $\varepsilon>0$, we have

$$
\begin{aligned}
& \int_{a_{\rho} x}^{\left(a_{\rho}+\varepsilon\right) x} \boldsymbol{P}\{W>x / r, z(W)>a x / r\} d F(r) \\
& =\int_{0}^{\varepsilon v\left(x_{*}\right)} \boldsymbol{P}\left\{W>1 / a_{\rho}\left[1+s / v\left(x_{*}\right)\right]^{-1}, z(W)>a / a_{\rho}\left[1+s / v\left(x_{*}\right)\right]^{-1}\right\} d F_{x}(s) \\
& =\int_{0}^{\varepsilon v\left(x_{*}\right)} \boldsymbol{P}\left\{W>1 / a_{\rho}\left[1+s / v\left(x_{*}\right)\right]^{-1}, W<z_{\varepsilon}\left(a / a_{\rho}\left[1+s / v\left(x_{*}\right)\right]^{-1}\right)\right\} d F_{x}(s) \\
& =\int_{0}^{\varepsilon v\left(x_{*}\right)} \boldsymbol{P}^{*}\left\{\operatorname{cas} /\left(a_{\rho} v\left(x_{*}\right)\right)(1+o(1))>W-1 / a_{\rho}>-s /\left(a_{\rho} v\left(x_{*}\right)\right)(1+o(1))\right\} d F_{x}(s) .
\end{aligned}
$$

Hence by the assumptions on $W$ and $F$ applying Potter's bound for the integrand and utilising Lemmas 7.5 and 7.7 in Hashorva (2007) we obtain

$$
\begin{aligned}
& \int_{a_{\rho} x}^{\left(a_{\rho}+\varepsilon\right) x} \boldsymbol{P}\{W>x / r, z(W)>a x / r\} d F(r) \\
& \sim \int_{0}^{\infty} s^{\gamma_{a}} \exp (-s) d s L_{-1 / a_{\rho}, c a / a_{\rho}}\left(1 / v\left(x_{*}\right)\right) \frac{\bar{F}\left(x_{*}\right)}{\left(v\left(x_{*}\right)\right)^{\gamma a}} .
\end{aligned}
$$

In view of Eq. $37 a_{\rho}$ is necessarily unique, hence applying Eq. 14 we obtain as $x \rightarrow \infty$

$$
\begin{aligned}
p_{a}(x)= & \bar{F}(a / \rho)+(1+o(1)) \boldsymbol{P}\left\{I_{1}=I_{2}=1\right\} \Gamma\left(\gamma_{a}+1\right) L_{-1 / a_{\rho}, c a / a_{\rho}}\left(1 / v\left(x_{*}\right)\right) \\
& \times \frac{\bar{F}\left(x_{*}\right)}{\left(v\left(x_{*}\right)\right)^{\gamma_{a}}}+O\left(\bar{F}\left(\left(a_{\rho}+\varepsilon\right) x\right)\right) \\
\sim & \boldsymbol{P}\left\{I_{1}=I_{2}=1\right\} \Gamma\left(\gamma_{a}+1\right) L_{-1 / a_{\rho}, c a / a_{\rho}}\left(1 / v\left(x_{*}\right)\right) \frac{\bar{F}\left(x_{*}\right)}{\left(v\left(x_{*}\right)\right)^{\gamma a}},
\end{aligned}
$$

and thus the result follows.

Proof of Theorem 3 By the assumption on the density function $h$ of $W$ we have that Eq. 23 holds for any $K_{1}<K_{2}, K_{1}, K_{2} \in \mathbb{R}$ with $\gamma_{a}=1$. As in the proof above for any $\delta \in[0, \infty)$ and $\varepsilon>0$ small enough we obtain $($ set $\xi:=(c a \eta+\delta) /(c a+1))$

$$
\begin{aligned}
p_{a, \delta, \eta, \rho}(x)= & (1+o(1)) \boldsymbol{P}\left\{I_{1}=I_{2}=1\right\} \\
& \times \int_{\xi}^{\varepsilon v\left(x_{*}\right)} \boldsymbol{P}\left\{\frac{(1+o(1)) c a(s-\eta)}{a_{\rho} v\left(x_{*}\right)}>W-1 / a_{\rho}>\frac{(1+o(1))(\delta-s)}{a_{\rho} v\left(x_{*}\right)}\right\} d F_{x}(s) \\
\sim & \boldsymbol{P}\left\{I_{1}=I_{2}=1\right\} \int_{\xi}^{\infty}[(c a+1) s-\delta-\eta] \exp (-s) d s \frac{h\left(1 / a_{\rho}\right)}{a_{\rho}} \frac{\bar{F}\left(x_{*}\right)}{v\left(x_{*}\right)}, x \rightarrow \infty,
\end{aligned}
$$

hence the result follows. 
Acknowledgments I would like to thank both referees of the paper for very kind and deep review which improved the presentation substantially, and Professor Qihe Tang for inspiring related co-research.

\section{References}

Berman, M.S.: A low of large numbers for the maximum in a stationary Gaussian sequence. Ann. Math. Stat. 33(1), 93-97 (1962)

Berman, M.S.: Sojourns and extremes of Fourier sums and series with random coefficients. Stoch. Process. Their Appl. 15, 213-238 (1983)

Bingham, N.N., Goldie, C.M., Teugels, J.: Regular Variation. Cambridge University Press, Cambridge (1987)

Cambanis, S., Huang, S., Simons, G.: On the theory of elliptically contoured distributions. J. Multivar. Anal. 11(3), 368-385 (1981)

Dai, M., Mukherjea, A.: Identification of the parameters of a multivariate normal vector by the distribution of the minimum. J. Theor. Probab. 14(1), 267-298 (2001)

Das, B., Resnick, S.I.: Detecting a conditional extreme value model. Extremes (2011, in press)

Davis, R.A., Resnick, S.I.: Extremes of moving averages of random variables from the domain of attraction of the double exponential distribution. Stoch. Process. Their Appl. 30, 41-68 (1988)

De Haan, L., Ferreira, A.: Extreme Value Theory. An Introduction. Springer, Berlin (2006)

De Haan, L., Neves, C., Peng, L.: Parametric tail copula estimation and model testing. J. Multivar. Anal. 99, 1260-1275 (2008)

Embrechts, P., Klüppelberg, C., Mikosch, T.: Modelling Extremal Events for Insurance and Finance. Springer, Berlin (1997)

Falk, M., Hüsler, J., Reiss, R.-D.: Laws of small numbers: extremes and rare events. In: DMV Seminar, vol. 23, 3rd edn. Birkhäuser, Basel (2010)

Fang, K.-T., Fang, B.-Q.: Generalised symmetrised Dirichlet distributions. In: Fang, K.T., Anderson, T.W. (eds.) Statistical Inference in Elliptically Contoured and Related Distributions, pp. 127-136. Allerton, New York (1990)

Hashorva, E.: Asymptotic properties of type I elliptical random vectors. Extremes 10(4), 175-206 (2007)

Hashorva, E.: Conditional limiting results for Type I polar distributions. Extremes, 12(3), 239-263 (2009a)

Hashorva, E.: Conditional limits of $W_{p}$ scale mixture distributions. J. Stat. Plan. Inference 139(10), 3501$3511(2009 b)$

Hashorva, E.: Exact tail asymptotics of Dirichlet distributions (2009c). arXiv:0904.0144v1

Hashorva, E.: On the residual dependence index of elliptical distributions. Stat. Probab. Lett. 80(13-14), 1070-1078 (2010)

Hashorva, E., Hüsler, J.: Extreme values in FGM random sequences. J. Multivar. Anal. 68(2), 212-225 (1999)

Hashorva, E., Pakes, A.G., Tang, Q.: Asymptotics of random contractions. Insur., Math. Econ. 47(3), 405-414 (2010)

Hüsler, J., Li, D.: Testing asymptotic independece in bivarite extremes. J. Stat. Plan. Inference 139, 990998 (2009)

Haug, S., Klüppelberg, C., Peng, L.: Statistical models and methods for dependence in insurance data. J. Korean Stat. Soc. (2011, to appear)

Jessen, A.H., Mikosch, T.: Regularly varying functions. Publ. Inst. Math. (Nouvelle Série) 80(94), 171-192 (2006)

Lu, D., Li, W.V.: A note on multivariate Gaussian estimates. J. Math. Anal. Appl. 354(2), 704-707 (2009)

Li, D., Peng, L.: Goodness-of-fit test for tail copulas modeled by elliptical copulas. Stat. Probab. Lett. 79, 1097-1104 (2009)

Peng, L.: Second Order Condition and Extreme Value Theory. Tinbergen Institute Research Series 178. Thesis Publisher, Amsterdam (1998)

Peng, L.: Estimating the probability of a rare event via elliptical copulas. NAAJ, 12(2), 116-128 (2008)

Peng, L.: A practical way for estimating tail dependence functions. Stat. Sin. 20, 365-378 (2010)

Peng, L., Qi, Y.: Smoothed jackknife empirical likelihood method for tail copulas. Test (2011, to appear)

Manner, H., Segers, J.: Tails of correlation mixtures of elliptical copulas. Insur., Math. Econ. 48, 153-160 (2011) 
Reiss, R.-D.: Approximate Distributions of Order Statistics: With Applications to Nonparametric Statistics. Springer, New York (1989)

Reiss, R.-D., Thomas, M.: Statistical Analysis of Extreme Values. From Insurance, Finance, Hydrology and Other Fields, 3rd edn. Birkhäuser, Basel (2007)

Resnick, S.I.: Extreme Values, Regular Variation and Point Processes, Soft Cover Edition. Springer, New York (2008) 